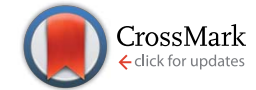

Cite this: RSC Adv., 2015, 5, 25616

\title{
Antireflective gradient-refractive-index material- distributed microstructures with high haze and superhydrophilicity for silicon-based optoelectronic applications
}

\begin{abstract}
Minkyu Choi, Jung Woo Leem and Jae Su Yu*
We fabricate gradient-index $(n)$ material-based microstructures, i.e., magnesium fluoride $\left(\mathrm{MgF}_{2}, n \sim 1.37\right)$ film-coated SU8 ultraviolet curable polymer ( $n \sim 1.59$ ) microcones (MCs) with tapered architectures, on silicon ( $\mathrm{Si}, n \sim 3.9$ ) substrates using a soft imprint lithography method for high-performance Si-based optoelectronic applications. The effects of various geometry parameters (i.e., height, filling ratio, and period) of conical MCs on the SU8 film/Si structure including different thicknesses of $\mathrm{MgF}_{2}$ films on the reflectance properties are investigated by a theoretical analysis using a rigorous coupled-wave analysis simulation. For the fabricated samples, their optical characteristics and surface wetting behaviors are also explored. The $\mathrm{MgF}_{2}$ film-coated SU8 $\mathrm{MCs}$ on $\mathrm{Si}$ substrates (i.e., $\mathrm{MgF}_{2} / \mathrm{SU} 8 \mathrm{MCs} / \mathrm{Si}$ ) exhibit a superhydrophilic surface with very low water contact angles of $<10^{\circ}$ and reduce the surface reflection of the bare Si over a wide wavelength of 350-1100 nm, showing the lower average reflectance $\left(R_{\text {avg }}\right)$ and solar weighted reflectance $\left(R_{\mathrm{sw}}\right)$ values of $\sim 14 \%$ and $\sim 12.1 \%$, respectively, (i.e., $R_{\text {avg }} \sim 15.1 \%$ and $R_{\mathrm{sw}} \sim$ $13 \%$ for the SU8 MCs/Si and $R_{\text {avg }} \sim 38.5 \%$ and $R_{\mathrm{sw}} \sim 38 \%$ for the bare Si). Furthermore, the $\mathrm{MgF}_{2} / \mathrm{SU} 8$ MCs on glasses also show strong light scattering in transmissions at wavelengths of 350-1100 nm, which indicates an average haze ratio of $\sim 89.8 \%$, while maintaining high total transmissions. Both the measured and calculated reflectance spectra showed similar results.
\end{abstract}

Received 3rd December 2014 Accepted 2nd March 2015

DOI: $10.1039 / c 4 r a 15686 b$

www.rsc.org/advances complex nanolithography techniques, e.g., laser interference, ${ }^{14,15}$ electron (e)-beam, ${ }^{16}$ and nanoimprint ${ }^{17}$ lithography methods and thermal ${ }^{18}$ and electrochemical ${ }^{19}$ treatments, together with a dry etching are usually required. On the other hand, the AR microstructures can be easily fabricated using a cost-effective, simple, fast, and high-throughput soft imprint lithography method..$^{20-24}$ For conformable and elastomeric stamps, in most of reports on the soft lithography, polydimethylsiloxane (PDMS) with low free surface energy, flexibility, transparency, and hardness has been often used to transfer micro/nano patterns precisely on secondary substrates. ${ }^{20-24}$ For microstructured or nanostructured replicas, ultraviolet (UV) and thermally curable polymers have been utilized..$^{25-28}$ These polymers are suitable for ARCs on the surface of the Si due to relatively low refractive indices of $\sim 1.4-1.7$. In addition, the coverage of materials with a lower $n$ on the textured surface of higher $n$ materials would modify the refractive index profile, which further decreases the surface reflection due to the more gradient-refractive-index (GRIN) profile in terms of both the structures and materials. ${ }^{29-31}$ Besides, the microscale-based structures with larger periods than wavelengths of incident light can extend the effective optical paths and promote the transmitted diffuse lights, while keeping high total transmission properties (i.e., high optical haze). ${ }^{13,31-33}$ This light
Department of Electronics and Radio Engineering, Kyung Hee University, 1732 Deogyeong-daero, Giheung-gu, Yongin-si, Gyeonggi-do 446-701, Republic of Korea. E-mail: jsyu@khu.ac.kr 
scattering effect can improve the light absorption in the optical and optoelectronic devices. ${ }^{33-35}$ Meanwhile, the superhydrophilicity makes water droplets to spread out evenly like a thin film on the surface. When a water droplet flows on the hydrophilic surface, it can squeeze into the space between the dusts and the surface, and then take the dusts away, i.e., selfcleaning. ${ }^{18,19,36}$ Also, it has many functions such as anti-fog, quick dry, and elimination of light scattering caused by the water droplets. This hydrophilicity can be enhanced if the roughness is sufficiently large. ${ }^{37,38}$ Our group has previously reported the antireflective GRIN material-distributed microstructures with a strong light scattering effect, together with an optical analysis. ${ }^{31}$ The micrograting structures were fabricated on the surface of transparent sapphires by conventional photolithography patterning and subsequent dry etching processes. As mentioned earlier, however, the realization of directly-patterned structures on the $\mathrm{Si}$ surface using these patterning and etching processes leads to the increase of the complexity and cost in the fabrication process. To avoid this problem, here, we proposed a novel architectural approach consisting of GRIN material-distributed microcone arrays, which effectively reduces the surface reflectivity of $\mathrm{Si}$ and enhances the light scattering in the $\mathrm{Si}$, fabricated by the soft imprint lithography using the micrograting structured sapphires as a master mold and PDMS stamps. Although the master molds with microstructures are also prepared by the lithography patterning and etching processes, once master molds and replica polymer stamps are made, they can be repeatedly employed for pattern transfers into secondary substrates. Furthermore, the large-scale fabrication techniques (i.e., roll-to-roll and roll-to-plate processes) of master molds and stamps have been developed in the soft imprint lithography, ${ }^{39,40}$ which would facilitate the mass-production for industry applications. In addition, it is difficult to optimize experimentally efficient antireflective GRIN material-distributed microstructures with a strong light scattering effect on the Si surface due to high cost and time-consumption. To verify their optical properties with optimizing the structure, it is also necessary to perform numerical modeling and optical calculations. Therefore, it is very meaningful to analyze the optical properties and wetting behaviors of the GRIN material-based microstructures on the Si surface. In this work, we reported the optical design, fabrication, and characterization (i.e., optical properties and surface wettability) of magnesium fluoride $\left(\mathrm{MgF}_{2}\right)$-coated SU8 polymer microstructures on the Si surface via the soft imprint lithography and subsequent e-beam evaporation. For optical analyses, numerical modeling and simulations were carried out using the rigorous coupled-wave analysis (RCWA) and finitedifference time-domain (FDTD) methods.

\section{Numerical modeling details and simulations}

\section{Effect of geometry parameters on reflectance properties}

Firstly, the effects of various geometry parameters, such as height, filling ratio, and period, of conical MCs on the SU8 film/
Si substrate on reflectance properties were investigated by the RCWA method using a commercial software (DiffractMOD, Rsoft Design Group). For the $\mathrm{MgF}_{2}$ film-coated SU8 MCs/Si structure (i.e., $\mathrm{MgF}_{2} / \mathrm{SU} 8 \mathrm{MCs} / \mathrm{Si}$ ), the reflection calculations were also performed as a function of $\mathrm{MgF}_{2}$ film thickness. Fig. 1 shows the (a) contour plots of variations of calculated reflectance spectra of the SU8 MCs/Si structure for different geometry parameters of (i) height, (ii) filling ratio, and (iii) period including the (iv) $\mathrm{MgF}_{2}$ film thickness of the $\mathrm{MgF}_{2} / \mathrm{SU} 8 \mathrm{MCs} / \mathrm{Si}$ structure and (b) average reflectance $\left(R_{\text {avg }}\right)$ values for the corresponding geometry parameters at wavelengths of 350-1100 $\mathrm{nm}$. The fixed values for geometry parameters and threedimensional (3D) scale-modified corresponding simulation models with 2D periodic hexagonal pattern arrays used in these calculations are also shown in the inset of Fig. 1(a) and (b), respectively. To design the numerical model, the geometry of the conical MCs was roughly represented in the Cartesian coordinate system by a scalar-valued function of three variables, $f(x, y, z)$, for simplicity. The shape of conical MCs can be roughly defined by the following equations: ${ }^{27}$

$$
\begin{aligned}
& r=R_{\mathrm{MCs}} \times\left[\left(H_{\mathrm{MCs}}-z\right) / H_{\mathrm{MCs}}\right]^{1 / O_{\mathrm{T}}} \text { and } \\
& x^{2}+y^{2}=r^{2},\left(0 \leq z \leq H_{\mathrm{MCs}}\right),
\end{aligned}
$$

where $r$ is the radius of the circle in $x-y$ plane, and $H_{\mathrm{MCs}}, R_{\mathrm{MCs}}$, and $O_{\mathrm{T}}$ are the height, the bottom radius, and the order of taper of MCs, respectively. For all the calculations, it was assumed that the $O_{\mathrm{T}}$ was set to be 1.5 and thicknesses of SU8 film and Si substrate were kept at $1 \mu \mathrm{m}$ and $500 \mu \mathrm{m}$, respectively. As shown in Fig. 1, the SU8 MCs considerably reduce the surface reflection of the bare Si due to the GRIN profiles in terms of structure (i.e., tapered cone shape) and constituent materials (i.e., air/SU8/Si). However, their reflection property is strongly dependent on the height and filling ratio (i.e., ratio of the bottom diameter of MCs to the period between MCs; $R_{\mathrm{BDP}}$ ) of MCs as well as the $\mathrm{MgF}_{2}$ film thickness while there is relatively no significant variation for the period. As shown in Fig. 1(a), furthermore, the strong oscillations in calculation reflectance spectra of SU8 MCs/Si structure are observed over a wide wavelength range of 350-1100 $\mathrm{nm}$ due to the constructive or destructive interference caused by multiple reflections of the light at interfaces of the air/SU8 and SU8/Si. For the height in the (i) of Fig. 1(b), the $R_{\text {avg }}$ value is gradually decreased from 15.7 to $12.3 \%$ with increasing the height of MCs from 0.5 to $5 \mu \mathrm{m}$. This is attributed to the longer optical path length and more GRIN profile between air and the SU8 film via the MCs at larger heights. ${ }^{8,15,41}$ For the filling ratio $\left(R_{\mathrm{BDP}}\right)$, the high reflection region (i.e., red part in the (ii) of Fig. 1(a)) of $>21 \%$ is reduced with increasing the $R_{\mathrm{BDP}}$ value, exhibiting the $R_{\text {avg }}$ values of $19.7 \%$ at $R_{\mathrm{BDP}}=0.1$ and $13.8 \%$ at $R_{\mathrm{BDP}}=1$, as shown in the (ii) in Fig. $1(\mathrm{~b})$. This means that even if the MCs are formed, there is relatively little effect on reflectance properties at lower filling ratios. ${ }^{14,42}$ Thus, to effectively suppress the reflection, the closely-packed MCs are required. On the other hand, for a period range of $2-5 \mu \mathrm{m}$ in the (iii) of Fig. 1(b), the $R_{\text {avg }}$ values are maintained at similar values of $14.5 \pm 0.4 \%$. At the period of $2 \mu \mathrm{m}$, the SU8 MCs/Si has the lowest $R_{\text {avg }}$ value of $14.1 \%$. Unfortunately, for the realization of 

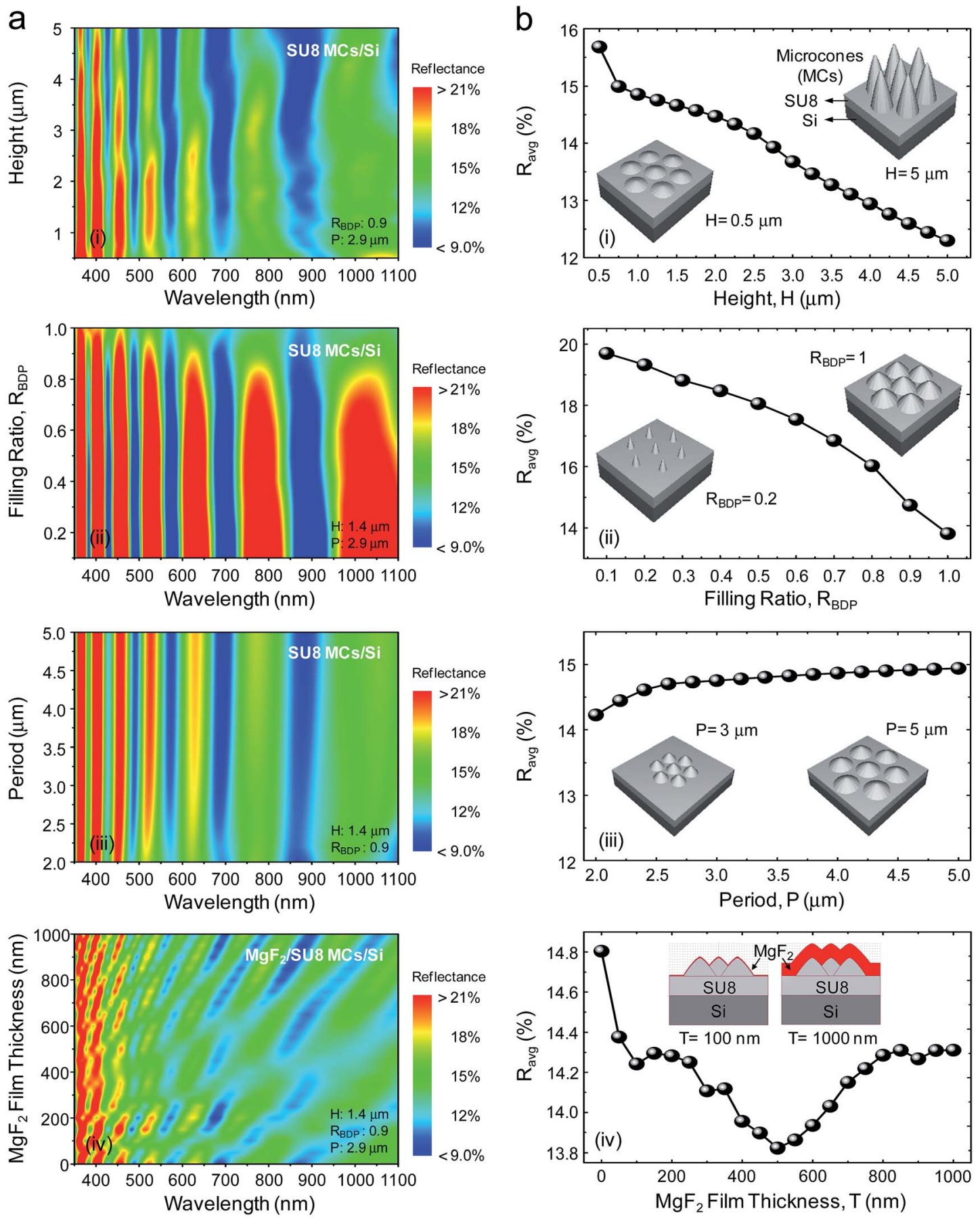

Fig. 1 (a) Contour plots of variations of calculated reflectance spectra of the SU8 MCs/Si structure for different geometry parameters of (i) height, (ii) filling ratio, and (iii) period including the (iv) $\mathrm{MgF}_{2}$ film thickness of the $\mathrm{MgF}_{2} / \mathrm{SU} 8 \mathrm{MCs} / \mathrm{Si}$ structure and (b) average reflectance $\left(R_{\text {avg }}\right)$ values for the corresponding geometry parameters at wavelengths of 350-1100 $\mathrm{nm}$. The fixed values for geometry parameters and three-dimensional (3D) scale-modified corresponding simulation models with 2D periodic hexagonal pattern arrays used in these calculations are also shown in the inset of (a) and (b), respectively.

MCs with small scale (i.e., sub-2 $\mu \mathrm{m}$ ), there are limitations in the conventional photolithography process such as the increase of fabrication cost and complexity on making soda lime or quartz mask substrates with sub-2 $\mu \mathrm{m}$ periods dotted metal (i.e., chrome) pattern arrays as well as the shorter photoresist (PR) development times, deformation/distortion of exposed PR patterns due to a interference caused by a UV-light diffraction in the short period mask patterns during the exposure process, and lower PR thicknesses on the formation of $2 \mathrm{D}$ hexagonal dotted PR pattern arrays with sub-2 $\mu \mathrm{m}$ periods on substrates. ${ }^{43,44}$ The MCs with sub- $2 \mu \mathrm{m}$ periods can be fabricated by other patterning techniques including laser 
interference, ${ }^{14,15}$ e-beam, ${ }^{16}$ or imprint ${ }^{17}$ lithography, colloidal formation, ${ }^{45,46}$ and thermally-dewetted metal particles. ${ }^{47,48}$ However, fabrication cost, simple approach, throughput, and process time of master molds as well as deformation and distortion of stamps and replicas pattern-transferred from the master molds with sub- $2 \mu \mathrm{m}$ scale features in the soft imprint lithography process should be considered for industrial applications. Lastly, the GRIN-material structure can further suppress the surface reflection. As can be seen in the (iv) of Fig. 1, the coating of $\mathrm{MgF}_{2}$ film with a lower $n$ onto the SU8 MCs/ Si further decreases the reflection, showing lower $R_{\text {avg }}$ values. Particularly, at a thickness of $500 \mathrm{~nm}$, the GRIN $\mathrm{MgF}_{2} / \mathrm{SU} 8 \mathrm{MCs} /$ Si structure has a $R_{\text {avg }}$ value of $13.8 \%$ which is lower than that (i.e., $R_{\text {avg }}=14.8 \%$ ) of SU8 MCs/Si without the $\mathrm{MgF}_{2}$ film. Thus, we deposited the $\mathrm{MgF}_{2}$ film with $500 \mathrm{~nm}$ of thickness on the SU8 $\mathrm{MCs} / \mathrm{Si}$ with geometry parameters including height of $1.4 \mu \mathrm{m}$, $R_{\mathrm{BDP}}$ of 0.9 , and period of $2.9 \mu \mathrm{m}$ in this experiment.

\section{Angle-dependent reflectance properties}

The conical micrograting structure can further suppress the surface reflection for the obliquely incident light due to its relatively omnidirectional shape. ${ }^{12}$ Fig. 2 shows the (a) contour plots of variations of calculated reflectance spectra of (i) the bare $\mathrm{Si}$, (ii) the SU8 $\mathrm{MCs} / \mathrm{Si}$, and (iii) the $\mathrm{MgF}_{2} / \mathrm{SU} 8 \mathrm{MCs} / \mathrm{Si}$ for different incident angles $\left(\theta_{\mathrm{i}}\right)$ of $0-80^{\circ}$ and (b) $R_{\text {avg }}$ values of the corresponding structures as a function of incident angle for non-polarized light. In Fig. 2, the incorporation of conical SU8 MCs into the surface of $\mathrm{Si}$ results in considerably reduced reflection in the wide ranges of wavelengths and incident angles. The SU8 MCs/Si structure has a much lower $R_{\text {avg }}$ value of $\sim 12.8 \%$ for $\theta_{\mathrm{i}}$ values of $0-80^{\circ}$ (i.e., $R_{\text {avg }} \sim 36.6 \%$ for the bare $\mathrm{Si}$ ). Meanwhile, the $\mathrm{MgF}_{2} / \mathrm{SU} 8 \mathrm{MCs} / \mathrm{Si}$ structure shows superior antireflective properties compared to the SU8 MCs/Si at $\theta_{\mathrm{i}}$ values of $<40^{\circ}$ while it exhibits slightly higher $R_{\text {avg }}$ values at some $\theta_{\mathrm{i}}$ values of $\geq 40^{\circ}$. This may be attributed to the variation of effective optical path lengths, which is related to the interferences in diffracted and rebounded lights at the interfaces of air/ $\mathrm{MgF}_{2}$ film, $\mathrm{MgF}_{2}$ film/SU8 MCs, and SU8 film/Si, caused by the change in the optical thickness of $\mathrm{MgF}_{2}$ film on the SU8 MCs/Si structure for larger oblique incident lights. ${ }^{49}$ Nevertheless, its $R_{\text {avg }}$ value of $\sim 12.4 \%$ for the entire $\theta_{\mathrm{i}}$ range of $0-80^{\circ}$ is lower than those of both the bare Si (i.e., $R_{\mathrm{avg}} \sim 36.6 \%$ ) and the SU8 $\mathrm{MCs} / \mathrm{Si}$ (i.e., $R_{\mathrm{avg}} \sim 12.8 \%$ ) samples. From these results, the conical microstructured polymer films can significantly suppress the surface reflection of $\mathrm{Si}$ over a wide range of wavelengths and incident angles. Moreover, the coating of materials with a lower $n$ on the SU8 MCs/Si can further reduce the reflection.

\section{Results and discussion}

\section{Fabrication and characterization of SU8 $\mathrm{MCs}$ and $\mathrm{MgF}_{2}$ film coating}

Fig. 3 shows the schematic diagram of process steps for the fabrication of $\mathrm{MgF}_{2}$ film-coated SU8 MCs on Si substrates via the soft lithography and e-beam evaporation. The scanning electron microscope (SEM) images of (i) sapphire master molds and (ii) PDMS stamps are also shown in Fig. 3. To form the MCs on SU8 polymer films, the patterned sapphire substrate, which was purchased from AND Corporation, consisting of conical MCs
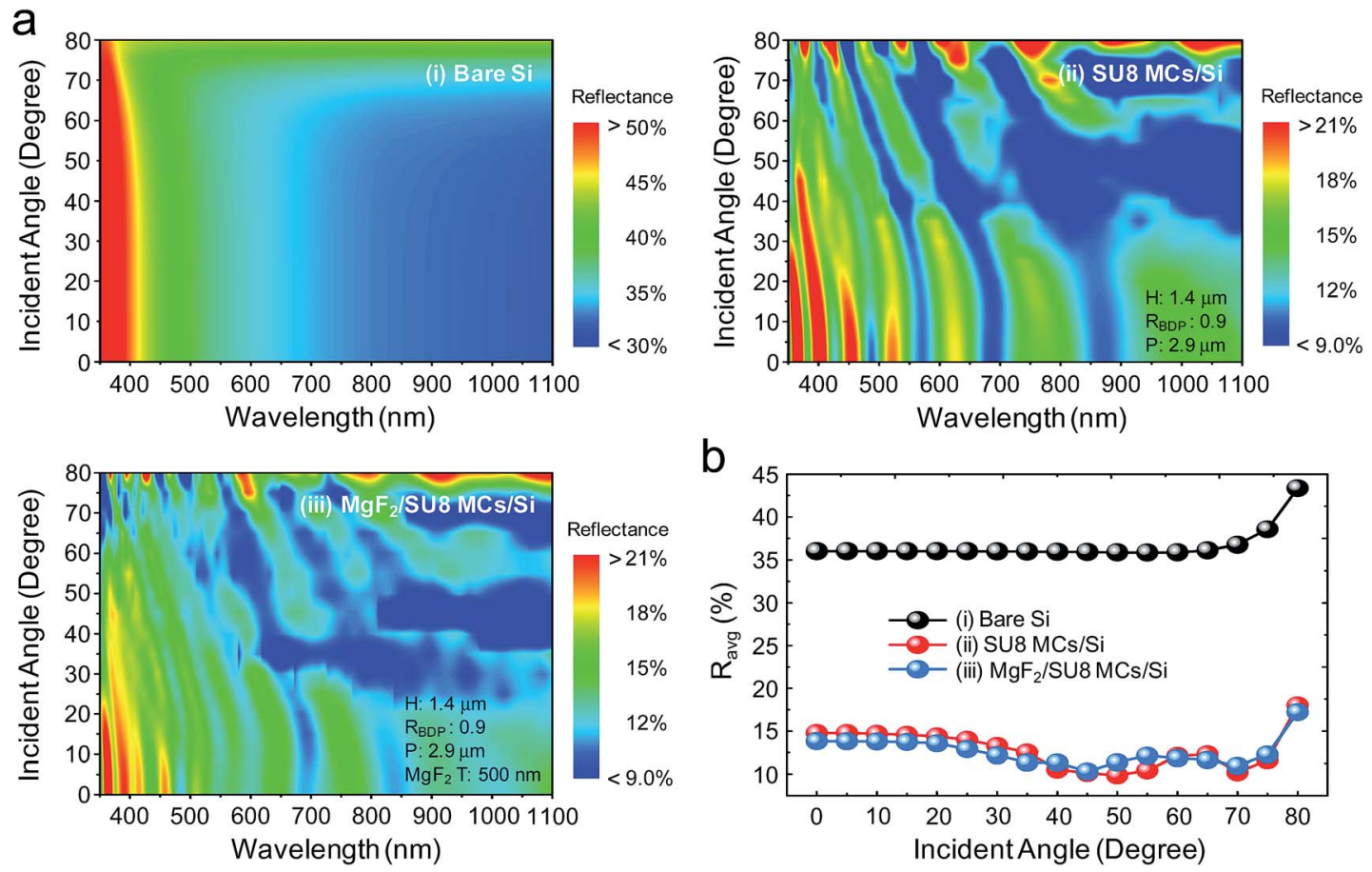

Fig. 2 (a) Contour plots of variations of calculated reflectance spectra of (i) the bare Si, (ii) the SU8 MCs/Si, and (iii) the MgF $2 / S U 8 \mathrm{MCs} / \mathrm{Si}$ for different incident angles $\left(\theta_{\mathrm{i}}\right)$ of $0-80^{\circ}$ and (b) $R_{\text {avg }}$ of the corresponding structures as a function of incident angle for non-polarized light. 


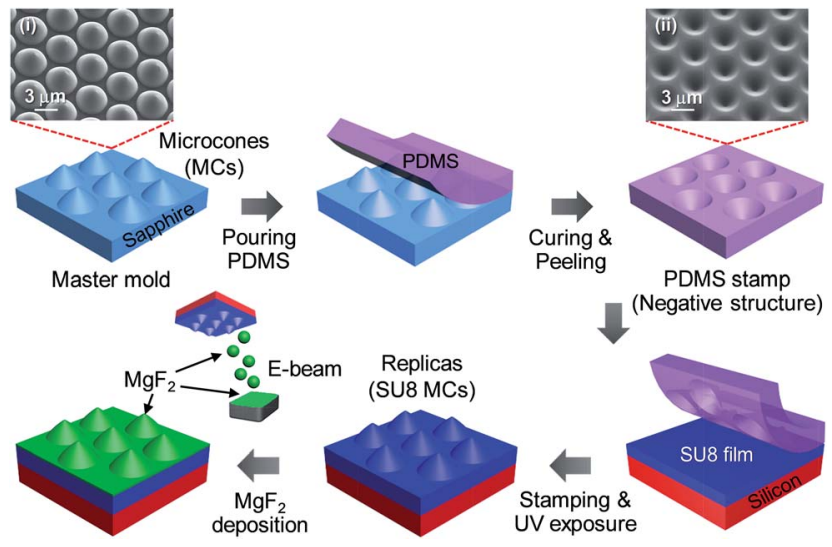

Fig. 3 Schematic diagram of process steps for the fabrication of $\mathrm{MgF}_{2}$ film-coated SU8 MCs on Si substrates via soft lithography and e-beam evaporation. The SEM images of (i) sapphire master molds and (ii) PDMS stamps are also shown, respectively.

with 2D periodic hexagonal patterns was used as a master mold, having average height and period of $\sim 1.5 \pm 0.1$ and $\sim 2.9 \pm 0.1$ $\mu \mathrm{m}$, as shown in the (i) SEM image of Fig. 3. In order to transfer MC patterns from the master mold into the Si substrate, Sylgard 184 (Dow Corning Co.) PDMS solution at a ratio of $10: 1$ (base : agent) was poured on master molds and then cured at a temperature of $75{ }^{\circ} \mathrm{C}$ for $2 \mathrm{~h}$. After that, the PDMS stamps were carefully separated from the molds, thus creating the negative conical MC patterned PDMS stamps, as shown in the (ii) SEM image of Fig. 3. The SU8 (Micro Chem Corp.) negative photoresist which is one of UV curable polymers was spin-coated on $\mathrm{Si}$ substrates with a size of $\sim 20 \mathrm{~mm} \times 20 \mathrm{~mm}$. The substrates were ultrasonically cleaned in acetone, methanol, and de-ionized water for $10 \mathrm{~min}$, respectively, and cured provisionally by pressing ( $3.0 \mathrm{kgf}$ ) the PDMS stamps. After UV exposure for 20 $\mathrm{min}$, the MCs on the surface of SU8 film/Si substrates were formed by peeling off the PDMS stamps. The $\mathrm{MgF}_{2}$ film was deposited on SU8 MCs/Si samples by using an e-beam evaporation system at room temperature with a rotation speed of 10 rpm. The deposition rate was kept at $2 \AA \mathrm{s}^{-1}$ using a quartz crystal sensor for thickness monitoring and rate control. The $\mathrm{MgF}_{2}$ film-coated SU8 MCs were also prepared on the soda lime float glass substrates (Microscope slides, Sail brand, Cat. no. 7101, manufactured by Yancheng Huida medical instruments Co., China).

The surface and cross-sectional morphologies of the samples were characterized by using a SEM (LEO SUPRA 55, Carl Zeiss) system. The reflectance and transmittance properties were characterized by using a UV-vis-NIR spectrophotometer (Cary 5000 , Varian) with an integrating sphere. The contact angles of water droplets were taken by using a contact angle measurement system (Phoenix-300, SEO Co., Ltd.) and averaged for three different positions on the surface of samples.

\section{Structural properties and surface wetting behaviors}

Fig. 4 shows (a) the $40^{\circ}$-tilted oblique- and side-view SEM images of the $\mathrm{MgF}_{2} / \mathrm{SU} 8 \mathrm{MCs} / \mathrm{Si}$ sample and (b) the photographs a

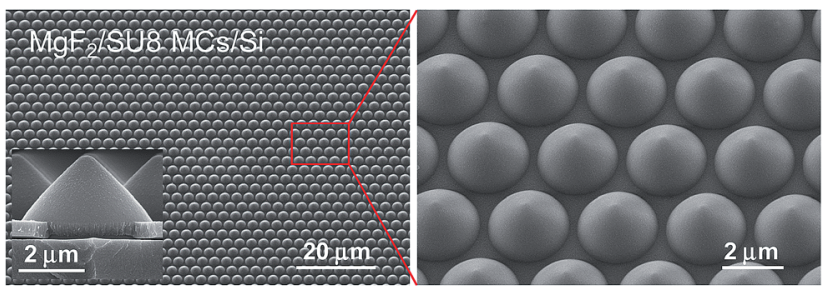

b

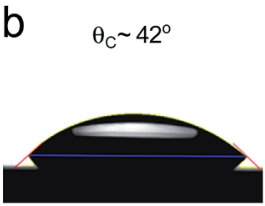

Bare Si

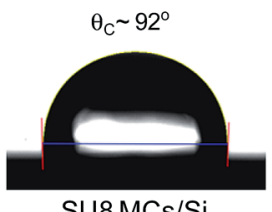

SU8 MCs/Si
$\theta_{\mathrm{C}}<10^{\circ}$

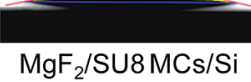

Fig. 4 (a) $40^{\circ}$-tilted oblique- and side-view SEM images of the $\mathrm{MgF}_{2}$ / SU8 MCs/Si sample and (b) photographs of a water droplet on the surface of the bare Si, the SU8 MCs/Si, and the $\mathrm{MgF}_{2} / \mathrm{SU} 8 \mathrm{MCs} / \mathrm{Si}$ samples.

of a water droplet on the surface of the bare $\mathrm{Si}$, the SU8 MCs/Si, and the $\mathrm{MgF}_{2} / \mathrm{SU} 8 \mathrm{MCs} / \mathrm{Si}$ samples. From the SEM images of Fig. 4(a), it can be observed that the conical MCs on SU8 film/Si substrates were well transferred from sapphire master molds via the PDMS stamps without any large deformation and distortion over a broad area. The $\mathrm{MgF}_{2}$ film with a thickness of $500 \mathrm{~nm}$ was also well deposited on the surface of SU8 MCs/Si samples. For the SU8 MCs, the average period and average height were also estimated to be $\sim 2.9 \pm 0.1$ and $1.4 \pm 0.1 \mu \mathrm{m}$, respectively. The filling ratio of MCs was about $R_{\mathrm{BDP}} \sim 0.9$.

The surface wetting properties strongly depend on the structures and materials. ${ }^{37,50}$ As shown in Fig. 4(b), the bare $\mathrm{Si}$ exhibited a hydrophilic surface with a water contact angle $\left(\theta_{\mathrm{C}}\right)$ of $\sim 42^{\circ}$. However, for the SU8 MCs, the $\theta_{\mathrm{C}}$ value of $\sim 92^{\circ}$ was obtained. This $\theta_{\mathrm{C}}$ value is higher than that $\left(\text { i.e., } \theta_{\mathrm{C}} \sim 74^{\circ}\right)^{26}$ of flat SU8 films without any patterns due to the increased roughness on the surface of SU8 films caused by the MCs, which can be explained by the Cassie-Baxter model. ${ }^{51}$ On the other hand, the $\mathrm{MgF}_{2}$ film-coated SU8 MCs/Si samples had very low $\theta_{\mathrm{C}}$ values of $<10^{\circ}$, indicating a superhydrophilic surface. This is due to the hydrophilic $\mathrm{MgF}_{2}$ film on the roughened MCs as proposed by the Wenzel's model. ${ }^{37}$ For the superhydrophilic surface, a water droplet can rapidly spread out and form a liquid film after falling onto the surface, and under the effect of gravity, the liquid film is easy to flow along the surface and takes dust particles or contaminants away. ${ }^{18,19,36}$ Therefore, these $\mathrm{MgF}_{2} /$ SU8 MCs/Si structures with a superhydrophilic surface may selfclean the pollutants and quickly dry the rainwater on the surface of Si-based optoelectronic devices in real outdoor environments. ${ }^{36,52}$

\section{Optical properties}

Measured reflectance spectra and $50^{\circ}$-titled oblique-view photographs of the bare $\mathrm{Si}$, the SU8 $\mathrm{MCs} / \mathrm{Si}$, and the $\mathrm{MgF}_{2} /$ SU8 MCs/Si samples are shown in Fig. 5(a) and (b), respectively. 
a

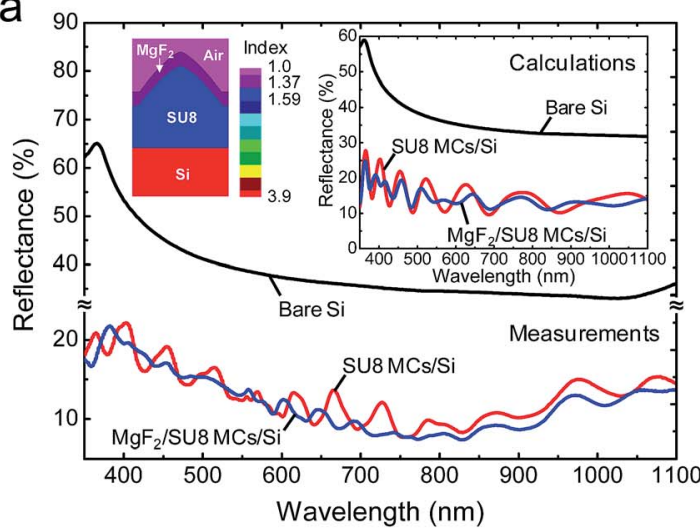

C

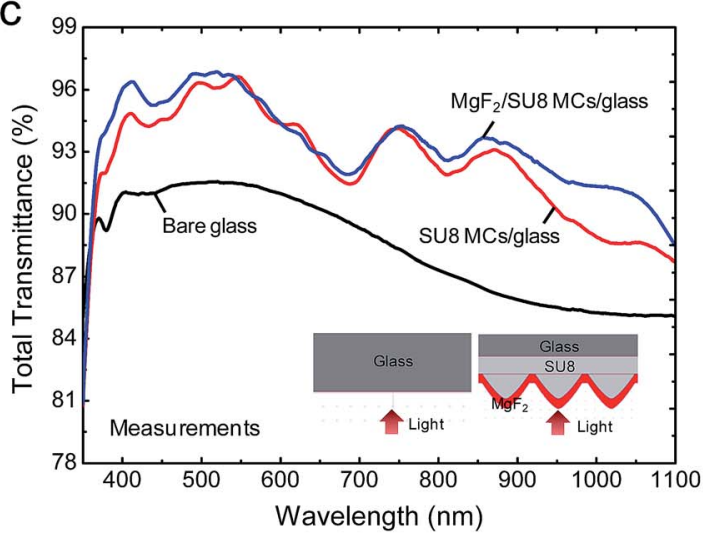

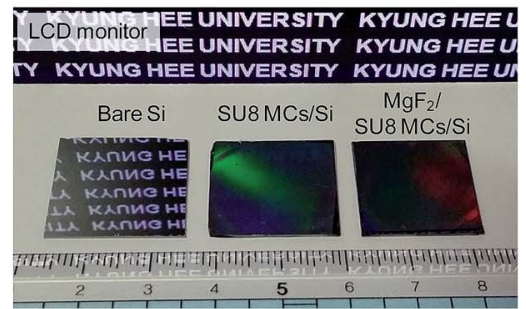

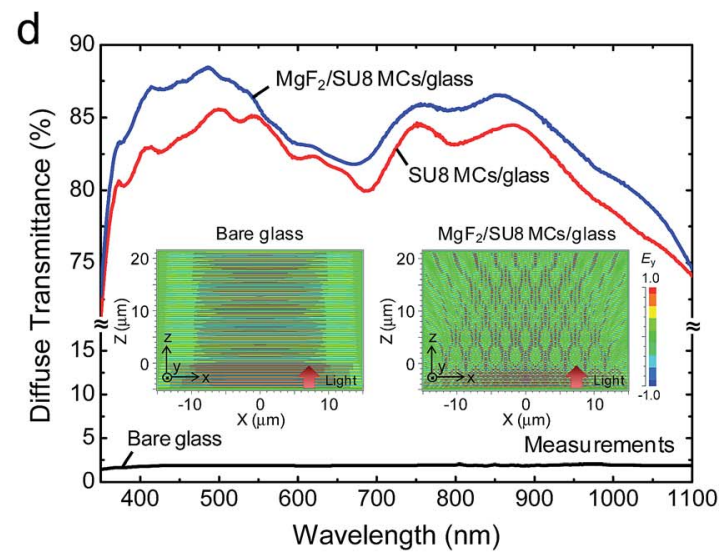

Fig. 5 (a) Measured reflectance spectra and (b) $50^{\circ}$-titled oblique-view photograph of the bare $\mathrm{Si}$, the $\mathrm{SU} 8 \mathrm{MCs} / \mathrm{Si}$, and the $\mathrm{MgF} / \mathrm{SU} 8 \mathrm{MCs} / \mathrm{Si}$ samples. Measured (c) total and (d) diffuse transmittance spectra of the bare glass, the SU8 MCs/glass, and the MgF $/ \mathrm{SU} 8 \mathrm{MCs} / \mathrm{glass}$ samples. The refractive index profile (left) of $\mathrm{MgF}_{2} / \mathrm{SU} 8 \mathrm{MCs} / \mathrm{Si}$ structure and calculated reflectance spectra (right) of the corresponding structures are also shown in the inset of (a). Contour plots of FDTD-calculated electric field $\left(E_{\mathrm{y}}\right)$ distributions for the incident light propagating from air to the bare glass and the $\mathrm{MgF}_{2} / \mathrm{SU} 8 \mathrm{MCs} /$ glass samples at $\lambda=620 \mathrm{~nm}$ are shown in the inset of (d).

The refractive index profile (left) of $\mathrm{MgF}_{2} / \mathrm{SU} 8 \mathrm{MCs} / \mathrm{Si}$ structure and calculated reflectance spectra (right) of the corresponding structures are also shown in the inset of Fig. 5(a). At wavelengths above $1030 \mathrm{~nm}$, the abrupt increase in reflectance spectra is attributed to the backscattered lights from the back surface of the Si substrate because $S i$ is transparent below its energy bandgap..$^{53}$ As shown in Fig. 5(a), the SU8 MCs considerably reduce the reflection, exhibiting a much lower $R_{\text {avg }}$ value of $\sim 15.1 \%$ than that of the bare $\mathrm{Si}\left(\right.$ i.e., $\left.R_{\text {avg }} \sim 38.5 \%\right)$. However, as confirmed in the calculated reflectance results in Fig. 1(d), the $500 \mathrm{~nm}$-thick $\mathrm{MgF}_{2}$ film-coated SU8 MCs/Si can further decrease the reflectance compared to the SU8 MCs/Si over a wide wavelength of $350-1100 \mathrm{~nm}$, showing the $R_{\text {avg }}$ value of $\sim 14 \%$. This is due to the formation of linear GRIN profile between air and the SU8 caused by the MCs and the step GRIN variation of the constituent materials from air $(n=1)$ to the $\mathrm{Si}(n$ 3.9) via the $\mathrm{MgF}_{2}(n \sim 1.37) / \mathrm{SU} 8(n \sim 1.59)$, which can be seen in the left inset of Fig. 5(a), as well as the extension of effective optical path lengths caused by the diffracted and rebounded lights between the MCs..$^{13,29,31-33,54,55} \mathrm{In}$ fact, the $\mathrm{MgF}_{2} / \mathrm{SU} 8 \mathrm{MCs} /$ Si with the $R_{\text {avg }} \sim 14 \%$ exhibits a relatively poor antireflection performance compared to other nanostructures or thin films as ARCs for reducing the surface reflectivity of the Si. The nanostructures including subwavelength-structured nipple grating or hollow-tip arrays dramatically reduced the surface reflectivity of the bare $\operatorname{Si}(R$ values of $>30 \%$ ) by $R$ values of $<5 \%$ in the wavelength range of $400-1100 \mathrm{~nm} \cdot{ }^{47,56-59}$ The thin film-based ARCs such as single layer (e.g., $\mathrm{SiN})^{60}$ or double layers (e.g., $\mathrm{SiO}_{2} /$ $\left.\mathrm{TiO}_{2}\right)^{61}$ coated on the $\mathrm{Si}$ also showed $R_{\text {avg }}$ values below than $10 \%$. However, most of nanostructures for ARCs have been usually realized by expensive and complicated fabrication processes including colloidal-sphere, ${ }^{56,57}$ nanoimprint, ${ }^{58}$ and laser interference ${ }^{59}$ lithography techniques as well as thermallydewetted metal nanoparticles ${ }^{47}$ with subsequent dry or chemical etching processes. On the other hand, the conventional ARCs consisting of a stack of thin-film dielectric layers can be fabricated by a relatively simple deposition or coating process. But, they also have some drawbacks such as poor or reduced substrate adhesion on certain materials due to the thermal expansion mismatch, sensitivity to thickness variations, and material selection as well as narrow low reflection band for incident wavelengths and angles, ${ }^{5-7}$ which may not be suitable in practical harsh environments. On the contrary, as mentioned earlier, in terms of the fabrication process for SU8 MCs, the soft imprint lithography is compatible with continuous processing, which is advantageous for simple, low-cost, and high- 
throughput production compared to other nanopatterning methods for etch mask patterns and subsequent dry etching processes. ${ }^{20-24}$ Master molds and stamps are also reusable. Additionally, mass production is possible by large-scale fabrication techniques for polymer stamps. ${ }^{39,40}$ Moreover, this pattern transfer technique can be also employed on various substrates including semiconductors, metals, and transparent materials. For photovoltaic device applications, it is necessary to investigate the solar weighted reflectance $\left(R_{\mathrm{sw}}\right)$, which is defined by the ratio of the usable photons reflected to the total useable photons, of samples. The $R_{\mathrm{sw}}$ can be estimated by normalizing the reflectance and the terrestrial air mass 1.5 global spectra integrated over a wavelength range of 350-1100 nm. ${ }^{3}$ For the $\mathrm{MgF}_{2} / \mathrm{SU} 8 \mathrm{MCs} / \mathrm{Si}$ sample, the relatively lower $R_{\mathrm{sw}}$ value of $\sim 12.1 \%$ was obtained compared to the SU8 $\mathrm{MCs} / \mathrm{Si}\left(\right.$ i.e., $R_{\mathrm{sw}} \sim 13 \%$ ) as well as the bare $\mathrm{Si}\left(\right.$ i.e., $\left.R_{\mathrm{sw}} \sim 38 \%\right)$. In the inset of Fig. 5(a), the reflectance spectra calculated by the RCWA method roughly show a similar trend with the measured data in Fig. 5(a). Also, their antireflective properties can be observed from the photograph in Fig. 5(b). The characters in the LCD monitor were strongly reflected by the surface of the bare $\mathrm{Si}$. On the contrary, both the SU8 MCs/Si samples with and without the $\mathrm{MgF}_{2}$ film almost had a black surface due to their low reflectivity at visible wavelengths, as can be seen in Fig. 5(a). Also, rainbow (e.g., green or red)-like colors on their surfaces were shown due to the strong light diffraction property.

To investigate the diffuse light scattering of SU8 MCs, the corresponding structures were also prepared on the surface of glasses. Measured total and diffuse transmittance spectra of the bare glass, the SU8 $\mathrm{MCs} / \mathrm{glass}$, and the $\mathrm{MgF}_{2} / \mathrm{SU} 8 \mathrm{MCs} /$ glass samples are shown in Fig. 5(c) and (d), respectively. As shown in Fig. 5(c), both the SU8 MCs/glass and $\mathrm{MgF}_{2} / \mathrm{SU} 8 \mathrm{MCs} /$ glass samples exhibited higher total transmission spectra than that of the bare soda lime glass, which shows the similar optical behavior reported in the previously-published literature, ${ }^{\mathbf{2 8 , 6 2}}$ in the wide wavelength range of 350-1100 $\mathrm{nm}$, indicating larger average transmittance $\left(T_{\text {avg }}\right)$ and solar weighted transmittance $\left(T_{\mathrm{sw}}\right)$ values of $\sim 92.4 \%$ and $\sim 93 \%$ for the SU8 MCs/glass and $\sim 93.4 \%$ and $\sim 93.9 \%$ for the $\mathrm{MgF}_{2} / \mathrm{SU} 8 \mathrm{MCs} /$ glass, respectively, (i.e., $T_{\mathrm{avg}} \sim 88.3 \%$ and $T_{\mathrm{sw}} \sim 89.1 \%$ for the bare glass). Besides, for a periodic grating structure, when light enters into the grating with a period of $\Lambda$ at normal incidence, the angle of the transmitted diffraction waves, $\theta_{\mathrm{t}, m}$, in the $m$-th diffraction order is given by the well-known grating equation: ${ }^{63}$

$$
\sin \theta_{\mathrm{t}, m}=\frac{m \lambda}{\Lambda n},
$$

where $\lambda$ is the incident wavelength of light and $n$ is the refractive index of the incident medium. From this, the grating structure with periods larger than incident wavelengths generates higher order diffracted lights in transmission. Therefore, the conical structures with desirable microscale periods can result in high total and diffuse transmittances, simultaneously. ${ }^{13,29,31-33,54,55}$ As shown in Fig. 5(d), the SU8 MCs/glass and $\mathrm{MgF}_{2} / \mathrm{SU} 8 \mathrm{MCs} /$ glass samples had very high diffuse transmittance spectra over a wide wavelength region of 350-1100 $\mathrm{nm}$ while there exist almost no diffracted lights for the bare glass. Furthermore, the diffuse transmittance of $\mathrm{MgF}_{2} / \mathrm{SU} 8 \mathrm{MCs} /$ glass was slightly enhanced compared to the SU8 MCs/glass due to the lower $n$ value of the $\mathrm{MgF}_{2}$ film. ${ }^{29,31}$ The haze ratio $(H)$, which is defined by the ratio of the diffuse $\left(T_{\mathrm{d}}\right)$ to the total $\left(T_{\mathrm{t}}\right)$ transmission, i.e., $H(\%)=T_{\mathrm{d}} / T_{\mathrm{t}}$ $\times 100$, is often used to characterize the light scattering properties of a sample. For both the SU8 MCs/glass and $\mathrm{MgF}_{2} / \mathrm{SU} 8$ MCs/glass, the average $H\left(H_{\text {avg }}\right)$ values were estimated to be $\sim 88.4$ and $89.8 \%$, respectively, at wavelengths of $350-1100 \mathrm{~nm}$ (i.e., $H_{\text {avg }} \sim 3.8 \%$ for the bare glass). In the transmission, these high diffraction behaviors (i.e., light scattering) can be confirmed from the FDTD simulations. The contour plots of FDTD-calculated electric field $\left(E_{\mathrm{y}}\right)$ distributions for the incident light propagating from air to the bare glass and the $\mathrm{MgF}_{2} / \mathrm{SU} 8$ MCs/glass samples at $\lambda=620 \mathrm{~nm}$ are shown in the inset of Fig. 5(d). In FDTD simulations, the $E_{\mathrm{y}}$, i.e., amplitude of y-polarized electric field, was calculated for the incident plane wave with a slab mode beam profile which is normalized at $\lambda=620 \mathrm{~nm}$. The simulation results indicate that the SU8 MCs with a period of $2.9 \mu \mathrm{m}$ exhibit strong light interference patterns with a wide angular spread and help the light propagation across the interface between air and the SU8 while there are no scattering lights for the flat bare glass. ${ }^{3,31}$ From these results, the $\mathrm{MgF}_{2}$ film-coated SU8 MCs can effectively reduce the surface reflection of Si and they can lead to the strong light scattering inside the bulk $\mathrm{Si}$, which can be useful to enhance the optical performance in optical and optoelectronic devices.

\section{Conclusions}

The $\mathrm{MgF}_{2} / \mathrm{SU} 8 \mathrm{MCs} / \mathrm{Si}$ consisting of GRIN-material structures were fabricated by the soft imprint lithography using sapphire master molds with 2D periodic hexagonal conical micrograting pattern arrays. In comparison with both the SU8 MCs/Si and bare $\mathrm{Si}$, their antireflective properties were experimentally and theoretically investigated, including their surface wetting behaviours. The coating of $\mathrm{MgF}_{2}$ film on the SU8 MCs/Si structure led to the reduction of reflection as well as the enhancement of diffused lights in transmissions. The $\mathrm{MgF}_{2}$ / SU8 MCs/Si sample had a superhydrophilic surface (i.e., $\theta_{\mathrm{C}}$ values of $<10^{\circ}$ ) and exhibited lower $R_{\mathrm{avg}}$ and $R_{\mathrm{sw}}$ values of $\sim 14 \%$ and $\sim 12.1 \%$, respectively, at wavelengths of 350-1100 nm (i.e., $R_{\text {avg }} \sim 15.1 \%$ and $R_{\text {sw }} \sim 13 \%$ for the SU8 MCs/Si and $R_{\text {avg }} \sim$ $38.5 \%$ and $R_{\mathrm{sw}} \sim 38 \%$ for the bare $\mathrm{Si}$, respectively). Additionally, it showed strong light diffraction with a $H_{\text {avg }}$ value of $\sim 89.8 \%$ over a wide wavelength region of 350-1100 $\mathrm{nm}$. These results can provide a better insight into the broadband wide-angle antireflection microstructures, which can be easily prepared by the simple and inexpensive soft lithography, with selfcleaning functioned superhydrophilic and large diffuse light scattering properties for the fabrication of high-performance Sibased optoelectronic devices.

\section{Acknowledgements}

This work was supported by the National Research Foundation of Korea (NRF) grant funded by the Korea government (MSIP) (no. 2013-068407). 


\section{Notes and references}

1 Z. Huang, J. E. Carey, M. Liu, X. Guo, E. Mazur and J. C. Campbell, Appl. Phys. Lett., 2006, 89, 033506.

2 C. P. Liu, J. Xin, L. Wang, J. J. Song, A. Y. S. Lee and P. Ho, RSC Adv., 2014, 4, 34669.

3 J. W. Leem, M. Choi and J. S. Yu, ACS Appl. Mater. Interfaces, 2015, 7, 2349.

4 M. A. Green, J. Zhao, A. Wang, P. J. Reece and M. Gal, Nature, 2001, 412, 805.

5 S. M. Yang, Y. C. Hsieh and C. A. Jeng, J. Vac. Sci. Technol., A, 2009, 27, 336.

6 P. Lalanne and G. M. Morris, Proc. SPIE, 1996, 2776, 300.

7 N. Kadakia, S. Naczas, H. Bakhru and M. Huang, Appl. Phys. Lett., 2010, 97, 191912.

8 J. W. Leem, K. S. Chung and J. S. Yu, Curr. Appl. Phys., 2012, $12,291$.

9 J. W. Leem, J. S. Yu, D. H. Jun, J. Heo and W. K. Park, Sol. Energy Mater. Sol. Cells, 2014, 127, 43.

10 W. L. Min, B. Jiang and P. Jiang, Adv. Mater., 2008, 20, 3914.

11 K. Kintaka, J. Nishii, A. Mizutani, H. Kikuta and H. Nakano, Opt. Lett., 2001, 26, 1642.

12 J. W. Leem, Y. M. Song and J. S. Yu, Nanoscale, 2013, 5, 10455.

13 J. W. Leem, M. S. Kim and J. S. Yu, J. Opt. Soc. Am. B, 2013, 30, 1665.

$14 \mathrm{~J}$. W. Leem and J. S. Yu, Opt. Express, 2012, 20, A431.

15 Y. M. Song, S. J. Jang, J. S. Yu and Y. T. Lee, Small, 2010, 6, 984.

16 Y. Kanamori, M. Ishimori and K. Hane, IEEE Photonics Technol. Lett., 2002, 14, 1064.

17 J. Tommila, V. Polojärvi, A. Aho, A. Tukiainen, J. Viheriälä, J. Salmi, A. Schramm, J. M. Kontio, A. Turtiainen, T. Niemi and M. Guina, Sol. Energy Mater. Sol. Cells, 2010, 94, 1845.

18 L. K. Verma, M. Sakhuja, J. Son, A. J. Danner, H. Yang, H. C. Zeng and C. S. Bhatia, Renewable Energy, 2011, 36, 2489.

19 J. Son, L. K. Verma, A. J. Danner, C. S. Bhatia and H. Yang, Opt. Express, 2011, 19, A35.

20 D. Qin, Y. Xia and G. M. Whitesides, Nat. Protoc., 2010, 5, 491.

21 C. Y. Liu, K. J. Chen, D. W. Lin, C. Y. Lee, C. C. Lin, S. H. Chien, M. H. Shih, G. C. Chi, C. Y. Chang and H. C. Kuo, Opt. Express, 2014, 22, 4516.

22 G. Shao, J. Wu, Z. Cai and W. Wang, Sens. Actuators, A, 2012, 178, 230.

23 J. K. Choi, J. K. Jin, M. L. Jin, C. J. An and H. T. Jung, $R S C$ Adv., 2014, 4, 12302.

24 J. K. Y. Ong, D. Moore, J. Kane and R. F. Saraf, ACS Appl. Mater. Interfaces, 2014, 6, 14278.

25 J. Bian, X. Fu, J. Hu, Y. Cui, Z. Li, C. Yuan, H. Ge, W. D. Li and Y. Chen, $R S C A d v ., 2014$, 4, 22155.

26 S. H. Lee, J. W. Leem and J. S. Yu, Opt. Express, 2013, 21, 29298.

27 J. W. Leem, S. Kim, S. H. Lee, J. A. Rogers, E. Kim and J. S. Yu, Adv. Energy Mater., 2014, 4, 1301315.
28 J. W. Leem, X. Y. Guan, M. Choi and J. S. Yu, Sol. Energy Mater. Sol. Cells, 2015, 134, 45.

29 J. W. Leem, Y. M. Song and J. S. Yu, Opt. Express, 2011, 19, A1155.

30 Y. H. Ko and J. S. Yu, Opt. Express, 2011, 19, 297.

31 Y. H. Ko and J. S. Yu, Opt. Express, 2011, 19, 15574.

32 Y. P. Chen, C. H. Lee and L. A. Wang, Nanotechnology, 2011, 22, 215303.

33 B. Janthong, Y. Moriya, A. Hongsingthon, P. Sichanugrist and M. Konagi, Sol. Energy Mater. Sol. Cells, 2013, 119, 209.

34 S. J. Tark, M. G. Kang, S. Park, J. H. Jang, J. C. Lee, W. M. Kim, J. S. Lee and D. Kim, Curr. Appl. Phys., 2009, 9, 1318.

35 Y. Nasuno, N. Kohama, K. Nishimura, T. Hayakawa, H. Taniguchi and M. Shimizu, Appl. Phys. Lett., 2006, 88, 071909.

36 S. Nishmoto and B. Bhushan, RSC Adv., 2013, 3, 671.

37 R. N. Wenzel, Ind. Eng. Chem., 1936, 28, 988.

38 J. W. Leem, J. S. Yu, J. Heo, W. K. Park, J. H. Park, W. J. Cho and D. E. Kim, Sol. Energy Mater. Sol. Cells, 2014, 120, 555.

39 F. C. Krebs, Sol. Energy Mater. Sol. Cells, 2009, 93, 465.

40 S. H. Ahn and L. J. Guo, ACS Nano, 2009, 3, 2304.

41 S. A. Boden and D. M. Bagnall, Appl. Phys. Lett., 2008, 93, 133108.

42 J. W. Leem, Y. P. Kim and J. S. Yu, J. Opt. Soc. Am. B, 2012, 29, 357.

43 Y. Xiong, Z. Liu and X. Zhang, Appl. Phys. Lett., 2008, 93, 111116.

44 S. Okazaki, J. Vac. Sci. Technol., B: Microelectron. Nanometer Struct.-Process., Meas., Phenom., 1991, 9, 2829.

45 C. M. Hsu, S. T. Connor, M. X. Tang and Y. Cui, Appl. Phys. Lett., 2008, 93, 133109.

46 B. D. Park, J. W. Leem and J. S. Yu, Appl. Phys. B, 2011, 105, 335.

47 J. W. Leem and J. S. Yu, Thin Solid Films, 2011, 519, 3792.

48 J. W. Leem, J. S. Yu, Y. M. Song and Y. T. Lee, Phys. Status Solidi A, 2011, 208, 1902.

49 W. Zhou, M. Tao, L. Chen and H. Yang, J. Appl. Phys., 2007, 102, 103105.

50 J. A. Howarter and J. P. Youngblood, Macromol. Rapid Commun., 2008, 29, 455.

51 A. B. D. Cassie and S. Baxter, Trans. Faraday Soc., 1944, 40, 546.

52 J. Son, S. Kundu, L. K. Verma, M. Sakhuja, A. J. Danner, C. S. Bhatia and H. Yang, Sol. Energy Mater. Sol. Cells, 2012, 98, 46.

53 M. L. Kuo, D. J. Poxon, Y. S. Kim, F. W. Mont, J. K. Kim, E. F. Schubert and S. Y. Lin, Opt. Lett., 2008, 33, 2527.

54 Y. M. Song, G. C. Park, S. J. Jang, J. H. Ha, J. S. Yu and Y. T. Lee, Opt. Express, 2011, 19, A157.

55 Z. Zhao and M. A. Green, IEEE Trans. Electron Devices, 1991, 38, 1925.

56 C. H. Sun, P. Jiang and B. Jiang, Appl. Phys. Lett., 2008, 92, 061112.

57 Y. Li, J. Zhang, S. Zhu, H. Dong, Z. Wang, Z. Sun, J. Guo and B. Yang, J. Mater. Chem., 2009, 19, 1806. 
58 Z. Yu, H. Gao, W. Wu, H. Ge and S. Y. Chou, J. Vac. Sci. Technol., B: Microelectron. Nanometer Struct.-Process., Meas., Phenom., 2003, 21, 2874.

59 J. W. Leem, Y. M. Song, Y. T. Lee and J. S. Yu, Appl. Phys. B, 2010, 100, 891.
60 Y. J. Lee, D. S. Ruby, D. W. Peters, B. B. McKenzie and J. W. P. Hsu, Nano Lett., 2008, 8, 1501.

61 S. Y. Lien, D. S. Wuu, W. C. Yeh and J. C. Liu, Sol. Energy Mater. Sol. Cells, 2006, 90, 2710.

62 M. Rubin, Sol. Energy Mater., 1985, 12, 275.

63 E. Hecht, Optic, Addison Wesley, 4th edn, 2002, ch. 10. 